\title{
A Method for Isolating Ions in Quadrupole Ion Traps Using an Excitation Waveform Generated by Frequency Modulation and Mixing
}

\author{
Ryan T. Hilger, Robert E. Santini, Carl A. Luongo, Boone M. Prentice, \\ and Scott A. McLuckey \\ Department of Chemistry \\ Purdue University \\ West Lafayette, Indiana 47907-2084
}

\footnotetext{
*Address reprint requests to:

Dr. Scott A. McLuckey

560 Oval Drive

Department of Chemistry

Purdue University

West Lafayette, IN 47907-2084, USA

Phone: (765) 494-5270

Fax: (765) 494-0239

E-mail: mcluckey@purdue.edu
} 


\begin{abstract}
We describe a new method for isolating ions in quadrupole ion traps using an excitation waveform generated by mixing a broadband waveform generated by frequency modulation (FM) with a sine-wave at the secular frequency of the ion to be isolated. In terms of resolution and efficiency, the mixed FM method exhibits performance nearly identical to isolation using the apex of the Mathieu stability diagram. A disadvantage of the mixed FM method is that isolations require additional time relative to apex-based methods. This disadvantage is shared by other methods that involve application of multi-frequency waveforms such as stored waveform inverse Fourier transform (SWIFT). An advantage of the mixed FM technique (also shared with other tailored waveform approaches), is applicability to a much larger $\mathrm{m} / \mathrm{z}$ range than apex-based methods. Indeed, the mixed FM technique performs identically to SWIFT in many respects. While the mixed FM technique is not nearly as flexible as SWIFT in terms of the frequency content of the generated waveforms, the mixed FM technique is much simpler to implement as it requires only two function generators and a frequency mixer. Tuning important parameters of the waveform such as notch frequency, notch width, and excitation bandwidth is also facilitated with the mixed FM technique.
\end{abstract}

Keywords: ion isolation, frequency modulation, quadrupole ion trap, high mass ion 


\section{INTRODUCTION}

Tandem mass spectrometry ${ }^{1}$ (MS/MS) is the process of isolating precursor ions of a specific mass-to-charge ratio $(\mathrm{m} / \mathrm{z})$, subjecting them to some physiochemical process (e.g. collision induced dissociation, electron transfer dissociation), and measuring the masses of the product ions. MS/MS is extensively used because it allows for identification and structural characterization of analytes using mass spectrometry. MS/MS also increases specificity and improves detection limits because of the reduction in chemical noise that results when monitoring product ions. Isolation is a critical step in any MS/MS workflow as it ensures that product ions are generated from the desired precursor ion. An ideal isolation method preserves all of the target precursor ions and removes all of the untargeted ions. The extent to which these things are possible depends on many factors, such as the difference in $\mathrm{m} / \mathrm{z}$ between the target ion and the untargeted ion(s), the abundance of the target ion relative to the untargeted ion(s), the available time, and, of course, the isolation method.

Presently, the most extensively used ion isolation device in tandem mass spectrometry is the quadrupole mass filter ${ }^{2}$. Linear quadrupoles are also widely used as ion guides and as collision cells in many tandem mass spectrometer platforms. A linear quadrupole can be used to isolate ions in several ways. For example, mass filtering can be effected by appropriate application of radio frequency (RF) and DC potentials to the four rods. In this configuration, the range of $\mathrm{m} / \mathrm{z}$ that is transmitted by the quadrupole can be reduced to only those ions with $\mathrm{m} / \mathrm{z}$ values that fall within the tip of the Mathieu stability diagram. Ions with $\mathrm{m} / \mathrm{z}$ values outside the stable range are said to be 'filtered' out with the low $\mathrm{m} / \mathrm{z}$ and high $\mathrm{m} / \mathrm{z}$ ions being ejected in orthogonal directions. 
A disadvantage of the quadrupole mass filter is that, as the nominally stable $\mathrm{m} / \mathrm{z}$ range is decreased (i.e., resolution is increased by approaching the tip of the stability diagram more closely), the acceptance of the device for successful transmission decreases. Since incoming ions enter the device with a range of energies, positions, and angles, the decreased acceptance results in decreased transmission efficiency even for theoretically stable ions. One way to circumvent this problem is to trap and cool the ions in the quadrupole operated in the RF-only mode before applying the DC potentials that raise the precursor ions to the apex of the stability diagram. We refer to this process as apex isolation. ${ }^{3}$ The cooling narrows the position and velocity distributions such that fewer precursor ions are lost upon isolation. A disadvantage of apex isolation is that the trapping and cooling process requires more time than simply passing the ions through a quadrupole mass filter.

After ions have been trapped and cooled in a quadrupole, several methods other than apex isolation can be used to isolate a precursor ion. These methods exploit the fact that, under these conditions, ions have $\mathrm{m} / \mathrm{z}$ dependent resonant frequencies. Ions can therefore be selectively removed from the trap by applying a sinusoidal signal at the appropriate frequency across one pair of rods. Isolation can be performed by applying signals at all frequencies except for a narrow band centered on the resonant frequency of the target precursor ion. This scenario can be realized by passing a broadband waveform through a bandpass filter, which has been shown to be effective for isolating ions in a three-dimensional ion trap. ${ }^{4,5}$ An alternative approach for generating a tailored waveform for ion isolation is the method of stored waveform inverse Fourier transform (SWIFT). ${ }^{6,7}$ In this method, a waveform consisting of a series of discreet signals, closely spaced in frequency, is defined in the frequency domain. Signals with frequencies nearby the ion to be isolated are removed in order to create a notch. The resulting 
frequency domain waveform is then inverse Fourier transformed, creating a time domain waveform that can be played back using an arbitrary waveform generator. SWIFT provides greater flexibility than the use of a broad-band waveform with a single frequency filter. For example, with SWIFT, multiple notches can be defined, allowing for simultaneous isolation at multiple $\mathrm{m} / \mathrm{z}$ values. However, SWIFT requires relatively specialized equipment including software for defining the waveforms in the frequency domain and performing the inverse Fourier transform. Such software typically includes algorithms for optimizing the phases of the frequency components such that output power is maximized. The software must also be coupled to an arbitrary waveform generator used to play back the waveforms. Additionally, fine tuning of the SWIFT waveform can only be performed in the software. After each step of fine tuning, the waveform must be recompiled, inverse Fourier transformed, and downloaded to the arbitrary waveform generator for playback. Below we describe a method for isolation of ions trapped in a quadrupole that produces results similar to SWIFT, and, although it is less flexible (e.g. cannot define multiple notches), it is more straightforward to implement and fine tuning is as simple as turning a knob.

A waveform containing signals at multiple, closely-spaced frequencies can also be created using frequency modulation (FM). If a sinusoidal carrier signal with frequency $f_{c}$ is modulated such that its frequency varies from $f_{c}-f_{d}$ to $f_{c}+f_{d}\left(f_{d}\right.$ is called the frequency deviation) by a sinusoid with frequency $f_{m}$ (the modulation frequency), the result is a waveform containing signals at intervals of $f_{m}$ covering the frequency range $f_{c}-f_{d}$ to $f_{c}+f_{d}$. In order to create a notched waveform suitable for ion isolation, the FM signal is multiplied by another sinusoid with frequency $f_{n}$ using a frequency mixer. When two signals (frequencies $f_{1}$ and $f_{2}$ ) are multiplied using a frequency mixer, the output is a waveform containing components at 
frequencies $f_{1}+f_{2}$ and $f_{1}-f_{2}$. When the FM signal is multiplied by $f_{n}$ in this manner, the result is a waveform containing two frequency sidebands. The lower sideband contains signals at intervals of $f_{m}$ spanning the frequency range $f_{n}-\left(f_{c}+f_{d}\right)$ to $f_{n}-\left(f_{c}-f_{d}\right)$. The upper sideband contains signals at intervals of $f_{m}$ spanning the frequency range $f_{n}+\left(f_{c}-f_{d}\right)$ to $f_{n}+\left(f_{c}+f_{d}\right)$. The region between the two sidebands is the notch, and exists between $f_{n}-$ $\left(f_{c}-f_{d}\right)$ and $f_{n}+\left(f_{c}-f_{d}\right)$. Figure 1 illustrates the waveform generation process using hypothetical values for the various frequencies and Figure 2 shows a frequency spectrum of an actual waveform generated by our apparatus.

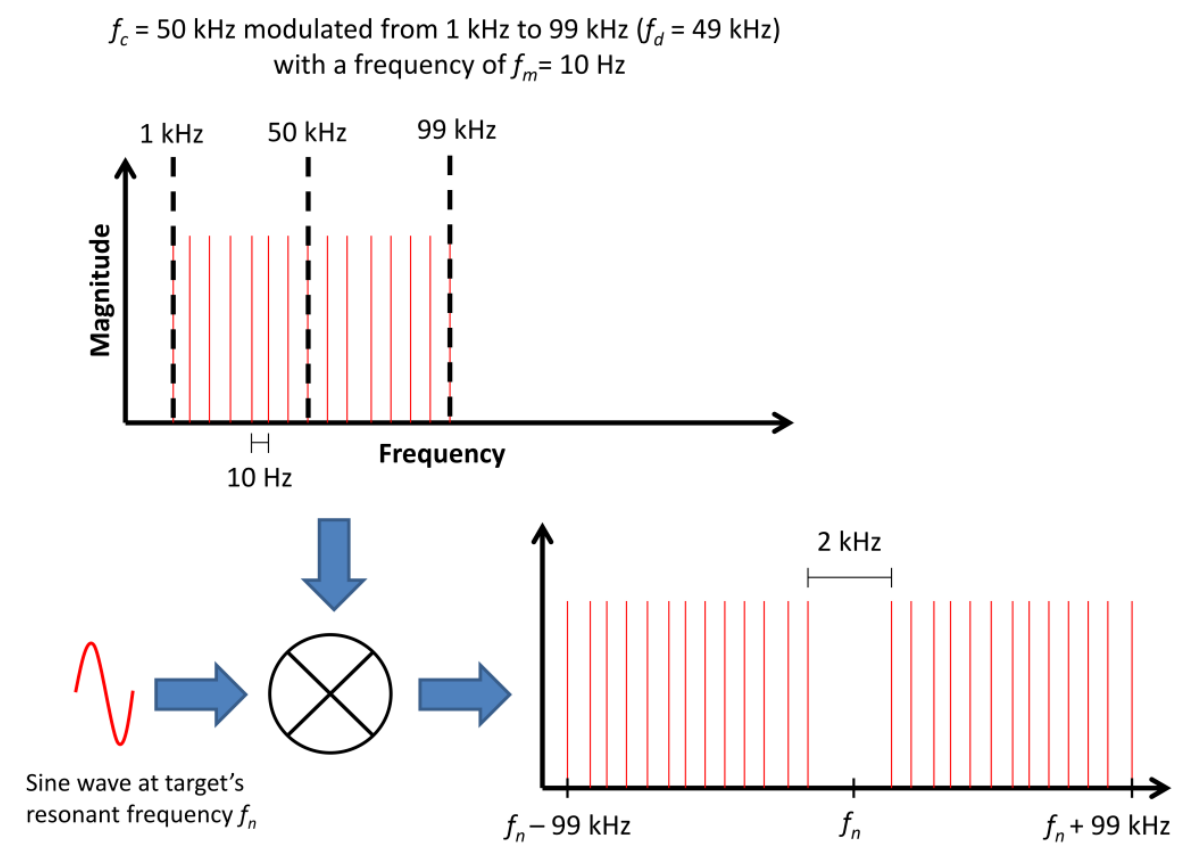

Figure 1. Cartoon illustrating generation of a notched broadband waveform using the mixed FM technique. The internal FM function of a function generator is used to produce a broadband waveform containing signals every $10 \mathrm{~Hz}$ over the range $1 \mathrm{kHz}$ to $99 \mathrm{kHz}$. This waveform is then mixed with a sine wave at frequency $f_{n}$ to produce a waveform with bands on either side of a 2 $\mathrm{kHz}$ wide notch. 

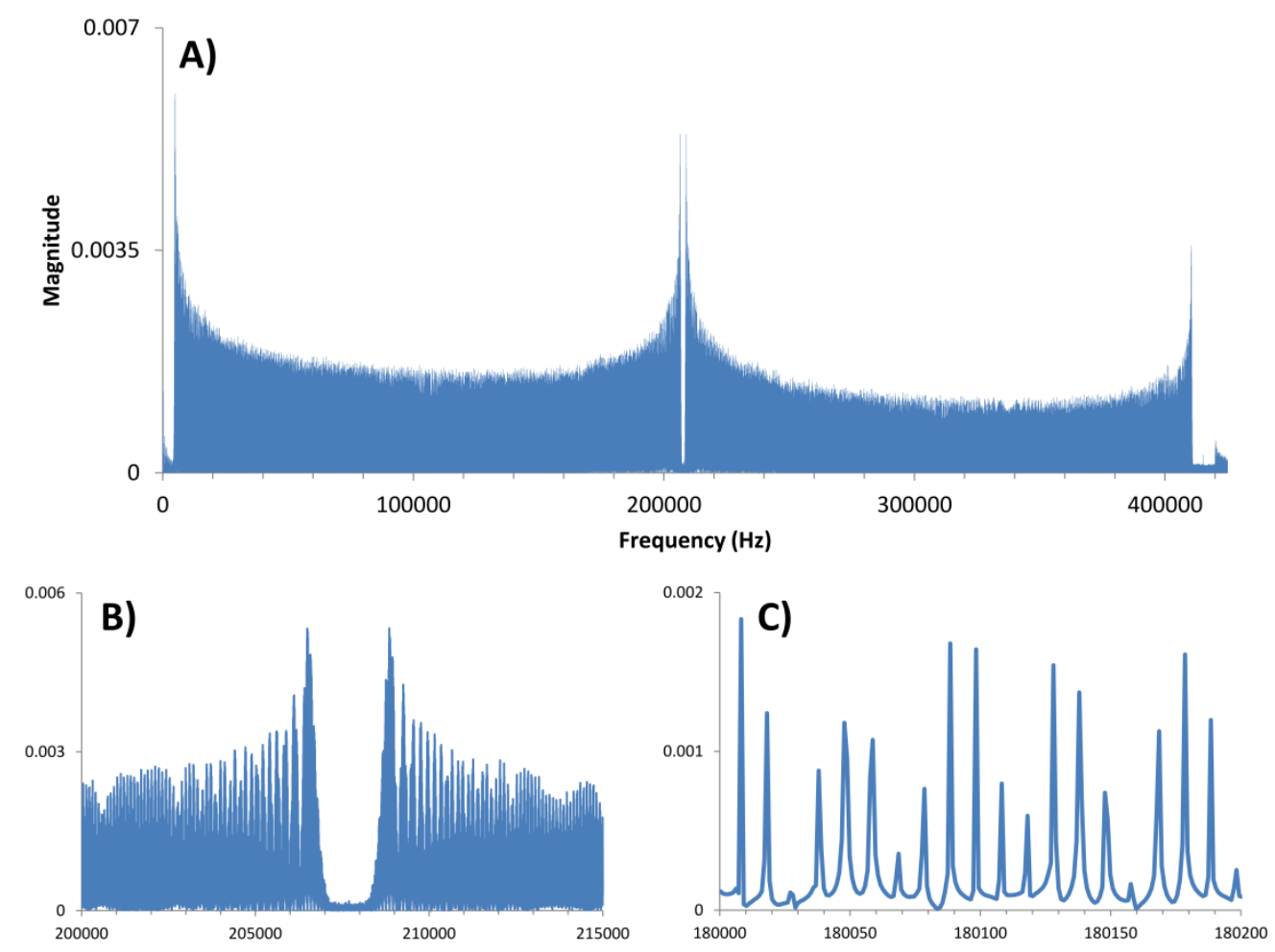

Figure 2. Fast Fourier transform of a mixed FM waveform captured by an oscilloscope. A) The frequency spectrum of the resulting waveform when $f_{c}=102 \mathrm{kHz}, f_{d}=101 \mathrm{kHz}, f_{n}=207.7$ $\mathrm{kHz}$, and $f_{m}=10 \mathrm{~Hz}$. The two sidebands and the notch are visible. B) Expanded view of the notch which is $2 \mathrm{kHz}$ wide and centered at $207.7 \mathrm{kHz}$. C) Expanded view showing the $10 \mathrm{~Hz}$ spacing of the signals within the lower sideband.

The above discussion reveals several important points. The waveform is centered on $f_{n}$. The bandwidth covered by the waveform is $2\left(f_{c}+f_{d}\right)$. The width of the notch (also centered on $\left.f_{n}\right)$ is $2\left(f_{c}-f_{d}\right)$. The frequency spacing of the signals making up the sidebands is $f_{m}$. Defining a waveform suitable for isolating ions in a quadrupole is straightforward. The notch frequency $f_{n}$ is chosen to be the fundamental secular (resonant) frequency of the ion to be isolated. The frequencies $f_{c}$ and $f_{d}$ are chosen such that the notch width provides the desired resolution and the bandwidth is sufficient to remove all untargeted ions. The modulation frequency $f_{m}$ must be sufficiently small such that the signals in the waveform are spaced closely enough to produce a quasi-continuum capable of removing untargeted ions at all frequencies within the bandwidth. 
The exact values chosen for $f_{n}, f_{c}, f_{d}$, and $f_{m}$ vary based on the ion to be isolated and the physical and electrical characteristics of the quadrupole. A more detailed discussion regarding determination of waveform parameters is provided below in the Results and Discussion section.

Waveform synthesis does not require a computer controlled arbitrary waveform generator, as is the case with SWIFT. The waveform described above can be synthesized using two function generators and a frequency mixer, which are often readily accessible, although it is a requirement that one function generator be capable of internal frequency modulation. An amplifier is typically also required to generate amplitudes capable of removing untargeted ions from the quadrupole, but this is true of all broadband frequency-based methods of isolating ions. Furthermore, fine tuning the mixed FM waveform is as simple as adjusting the settings on the two function generators. The parameter requiring the most frequent adjustment is the notch frequency $f_{n}$, since it must closely match the secular frequency of the ion of interest when performing high resolution isolations. Slight day-to-day drifts in ion secular frequencies make them difficult to calculate with sufficient precision. With the mixed FM technique, centering the notch on the ion of interest is achieved simply by turning a knob on the function generator. With SWIFT, the waveform must be redefined in the software and downloaded to the arbitrary waveform generator every time an adjustment is desired.

\section{EXPERIMENTAL}

Materials. ESI tuning mix for ion traps was purchased from Agilent Technologies (Santa Clara, CA). Methanol was purchased from Mallinckrodt (Phillipsburg, NJ). Polyethylene glycol $(\mathrm{PEG}$, average molecular weight $=3350 \mathrm{Da})$ was purchased from Sigma-Aldrich $($ St. Louis, MO). The salt of a carborane $\operatorname{acid}^{8}, \mathrm{Cs}\left(\mathrm{CHB}_{11} \mathrm{Cl}_{11}\right)$, was a generous gift from Prof. C. A. Reed of 
the Department of Chemistry, University of California at Riverside. The solution of PEG was prepared to $1 \mathrm{mg} / \mathrm{mL}$ in $50 / 50$ ( $\mathrm{vol} / \mathrm{vol})$ water/methanol. Carborane solution was prepared to 1 $\mathrm{mg} / \mathrm{mL}$ in water.

Mass Spectrometry. All experiments were performed on a QTRAP 4000 (AB Sciex, Toronto, ON) hybrid triple quadrupole linear ion trap mass spectrometer with modifications described previously. ${ }^{9}$ Sample was loaded into a pulled glass capillary and nanoelectrospray ionization was performed by applying a high potential to a platinum wire in contact with the solution. ${ }^{10}$ Ions were directed down a quadrupole ion guide (Q0) to Q1, which was operated either as an ion guide or as a mass filter. The ions then entered the collision cell (q2), where they were trapped and cooled by collisions with nitrogen gas before being transferred to Q3. The ions were then cooled in Q3 and, if necessary, isolation was performed using either the apex of the Mathieu stability diagram (apex isolation) or the mixed FM waveform. The isolated ions were then cooled before being analyzed using mass selective axial ejection (MSAE). ${ }^{11}$ Additional details regarding the isolations are given below alongside the data.

Ion/ion reactions were performed by sequentially injecting multiply sodiated PEG cations and singly charged carborane anions into the instrument via alternately pulsed nanoelectrospray. ${ }^{12}$ Carborane anions and $\left[\mathrm{PEG}(\mathrm{n}=72-80)+3 \mathrm{Na}^{+}\right]^{3+}$ cations were independently isolated in the Q1-mass filter prior to injection into the q2 mutual storage cell. The oppositely charged ions were allowed to react for $100 \mathrm{~ms}$ and subsequently transferred to Q3 for further isolation and mass analysis.

Generation and Application of Mixed FM Waveform. A block diagram of the setup used to generate and apply the mixed FM waveform is shown in Figure 3. The internal FM capability of function generator 1 (33220A, Agilent) was used to generate an FM signal 
containing frequency components spaced at intervals of $f_{m}=10 \mathrm{~Hz}$ spanning the frequency range $f_{c}-f_{d}$ to $f_{c}+f_{d}$ as described above. This signal was fed into the intermediate frequency (IF) port of a frequency mixer (ZAD-8+, Mini-Circuits, Brooklyn, NY). Fine tuning the DC offset of function generator 1 was necessary to eliminate signal breakthrough at $f_{n}$ at the output of the frequency mixer. Typically, a value of $-1.2 \mathrm{mV}$ was used for this DC offset. Function generator 2 (33120A, Agilent) was used to generate a sinusoid at the notch frequency $f_{n}$. This signal was fed into the local oscillator (LO) port of the frequency mixer. The output of the frequency mixer (radio frequency RF port) was connected to a home built amplifier (amp 1) based on an operational amplifier (PA09, Apex Microtechnology, Tucson, AZ) that provided a voltage gain of ten. The output of this amplifier was connected to a second home built amplifier (amp 2) based on the PA85 operational amplifier (Apex). The voltage gain of amp 2 was variable up to a maximum of twenty. The output of amp 2 was applied across one pair of Q3 rods using a transformer provided as a component of the mass spectrometer. This transformer was previously used to apply the dipolar excitation signal necessary for MSAE. Amp 2 also allowed for the application of a second signal (gain $=1$ ). The dipolar excitation signal was applied through this input in order to maintain MSAE functionality. Application of the mixed FM waveform was controlled by gating function generator 2. The amplitude of the mixed FM waveform was controlled either by tuning the output amplitude of function generator 2 (function generator 1 was maintained at an output amplitude of $300 \mathrm{mV}_{\mathrm{p}-\mathrm{p}}$, the maximum allowed by the frequency mixer) or by changing the gain of amp 2 . 


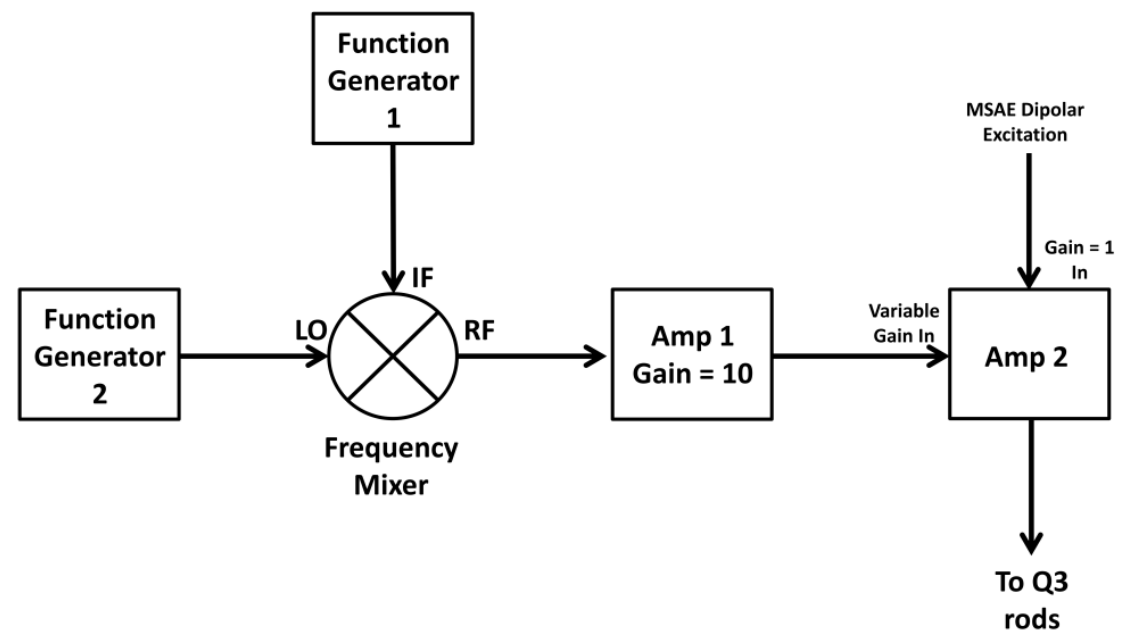

Figure 3. Block diagram of the apparatus for generating the mixed FM waveform and applying it to the Q3 rods. Provision was made for coupling in the dipolar excitation waveform required for MSAE.

\section{RESULTS AND DISCUSSION}

Defining the Mixed FM Waveform. In order to define the mixed FM waveform, the frequencies $f_{n}, f_{c}, f_{d}$, and $f_{m}$ must be chosen. A key consideration in these choices is the $q$ value of the ion of interest due to the fact that ion frequencies are determined by this value. When choosing a $q$-value for the ion to be isolated it is useful to bear in mind that the mixed FM waveform is symmetric about the notch. Therefore, it is convenient to position the ion to be isolated in the center of the frequency band occupied by ions within the quadrupole. The boundaries of the frequency band are determined by the points where the Mathieu stability diagram intercepts the $q$-axis at values of $q=0$ and $q=0.908$. The upper and lower frequency limits can be calculated (approximately) from ${ }^{13}$

$$
f_{\text {sec }}=\frac{f_{\text {drive }}}{2 \pi} \cos ^{-1}\left(1-\frac{1225-\left(35-\frac{2}{3}\left(\frac{\pi^{3} q}{16}\right)^{2}\right)^{2}}{70}\right)
$$


where $f_{\text {drive }}$ is the frequency of the RF potential applied to the quadrupole rods. For the quadrupole used in this work, $f_{\text {drive }}=816 \mathrm{kHz}$, which results in the ion fundamental secular frequencies $\left(f_{\text {sec }}\right)$ occupying the band from zero to $408 \mathrm{kHz}$. The midpoint is therefore $204 \mathrm{kHz}$, which corresponds to $q \approx 0.637$. The advantage of working at this $q$-value is that the bandwidth of the mixed FM waveform is minimized, since each sideband can be made to stretch exactly to the boundary of the ions' frequency band. At higher $q$-values, the target ion's secular frequency will be above the midpoint of the ions' frequency band, so the bandwidth must be expanded such that the lower sideband is able to reach the lower boundary of the band (zero frequency). In this case, the upper sideband extends to frequencies that are higher than those of any ions. At $q$ values below 0.637 , the bandwidth must also be expanded in order for the upper sideband to reach the upper boundary $(q=0.908)$. However, an interesting situation, referred to herein as the folding problem, occurs at $q=0.449$, as discussed below. Figure 4a shows a plot of the bandwidth required for the mixed FM waveform as a function of the $q$-value of the ion being isolated. 

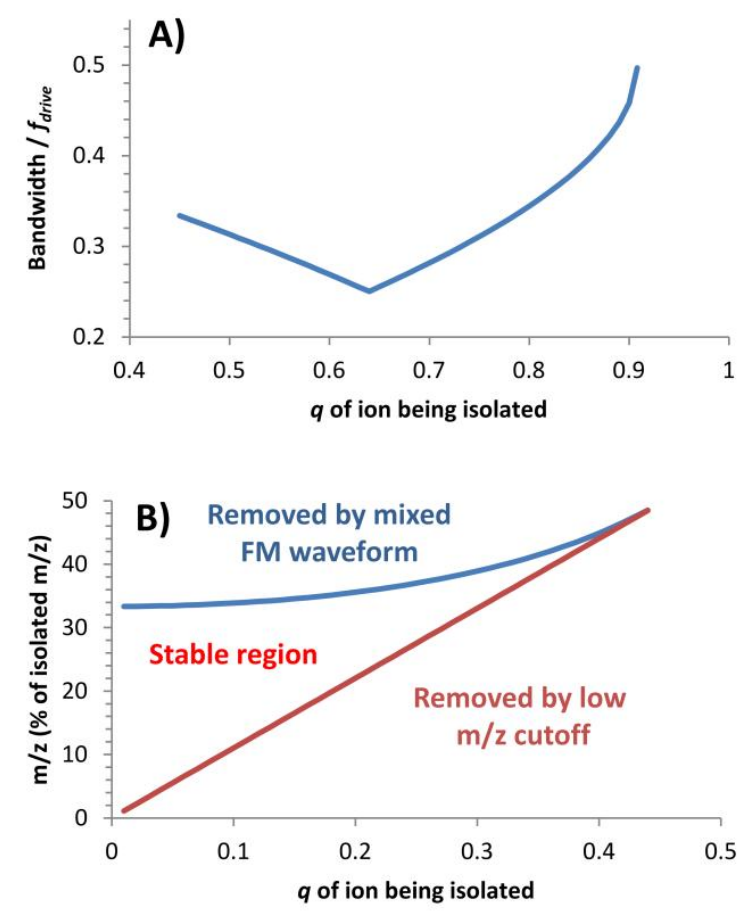

Figure 4. A) Bandwidth (expressed as a fraction of $f_{\text {drive }}$ ) required for the mixed FM waveform as a function of the $q$-value of the ion being isolated. The minimum bandwidth occurs at $q \approx$ 0.637. B) Minimum $\mathrm{m} / \mathrm{z}$ removed by the mixed FM waveform (blue line) and maximum $\mathrm{m} / \mathrm{z}$ removed by the low $\mathrm{m} / \mathrm{z}$ cutoff (red line) as a function of the $q$-value of the ion being isolated. Ions with $\mathrm{m} / \mathrm{z}$ between the red and blue lines will not be removed from the quadrupole during isolation.

Although $q=0.637$ allows for the lowest bandwidth of the mixed FM waveform, there may be situations in which it is desirable to choose $q$-values other than 0.637 . The $q$-value is adjusted by changing $V$, the amplitude of the RF potential applied to the rods, according to

$$
q=\frac{4 z e V}{m r_{0}^{2} \Omega^{2}}
$$

where $e$ is the electron's charge, $m$ is the mass of the ion, $r_{0}$ is the field radius of the quadrupole and $\Omega$ is the angular frequency of the RF potential applied to the rods. Changes to $q$ affect the pseudopotential well depth, $D$, according to 


$$
D=\frac{q V}{4}
$$

when $q \leq 0.4$. Larger well depths mean that the ions require more energy from the mixed FM waveform in order to be removed from the trap. At a given $q$-value, the well-depth increases with the $\mathrm{m} / \mathrm{z}$ of the ion because higher RF amplitudes are required for higher $\mathrm{m} / \mathrm{z}$ ions. For the equipment used in this work, we have found that, when high $\mathrm{m} / \mathrm{z}$ ions (above $\mathrm{m} / \mathrm{z}=1000$ ) are placed at high $q$-values (above 0.6), the output of our amplifiers was insufficient to remove them from the trap in a reasonable period of time (under one second).

What are the implications if the ion of interest is positioned at $q<0.637$ ? Under these conditions, the ion's secular frequency will be below the midpoint of the band. Therefore, the upper sideband of the mixed FM waveform must be extended in order to reach the upper frequency boundary. However, since the mixed FM waveform is symmetric, the lower sideband will be extended as well. The lower sideband will nominally pass through the boundary at $f=0$, which results in the nominally negative frequencies "folding" back across the $f=0$ line (i.e. a signal that would appear at $-10 \mathrm{kHz}$ appears at $+10 \mathrm{kHz}$ ). This does not cause a problem unless signals fold back into the notch and begin to obscure it. This occurs when the frequency corresponding to the lower boundary of the notch, $f_{n}-\left(f_{c}-f_{d}\right)$, is equal to twice the width of the sideband $\left(4 f_{d}\right.$ ). Typically, the width of the notch is variable but small compared to $f_{n}$. Therefore, for simplicity's sake, the lower boundary of the notch can be approximated to occur at $f_{n}$ (the notch is infinitely thin). Therefore, in order to avoid the folding problem the condition that 


$$
f_{d}<\frac{f_{n}}{4}
$$

should be met. In order for the upper sideband to reach the upper frequency boundary, $f_{d}$ must be equal to half of the difference between the upper frequency limit $\left(f_{\max }=f_{\text {drive }} / 2\right)$ and $f_{n}$

$$
f_{d}=\frac{f_{\max }-f_{n}}{2}
$$

Substituting equation 5) into equation 4) and solving for $f_{n}$ yields

$$
f_{n}>\frac{f_{\max }}{3}
$$

Equation 6 indicates that at values of $f_{n}$ below $f_{\max } / 3$, it is impossible to cover the ions' entire frequency band while preserving a notch at $f_{n}$, no matter how small. Equation 1 can be used to translate this lower frequency limit into a $q$-value, which turns out to be $q \approx 0.449$. When ions are positioned below this $q$-value, it is not possible to cover the entire frequency band with the mixed FM waveform, meaning that ions with frequencies very near the top end of the band ( $q$ approaching 0.908) will not be ejected from the trap. However, it is worth noting that, at high $q$ values ion frequency changes rapidly with $\mathrm{m} / \mathrm{z}$. Therefore, if the mixed FM waveform does not reach quite to the upper frequency boundary, the change in $\mathrm{m} / \mathrm{z}$ range may be quite small. For example, if the ion to be isolated is positioned at $q=0.4\left(f_{\text {sec }} \approx 120 \mathrm{kHz}\right)$, the upper sideband can only reach up to $q \approx 0.891\left(f_{\text {sec }}=360 \mathrm{kHz}\right)$. However, the $\mathrm{m} / \mathrm{z}$ range between $q=0.891$ and 0.908 amounts to less than $1 \%$ of the mass of the ion being isolated (i.e. less than $10 \mathrm{~m} / \mathrm{z}$ if 
$\mathrm{m} / \mathrm{z}=1000$ is being isolated), so the chance of a peak actually occupying this space is relatively small. Therefore, $q=0.4$ is a reasonable lower boundary with which one can be reasonably sure that all contaminating ions will be removed.

There are instances in which it may not be possible or desirable to position the ion of interest at a $q$-value in excess of 0.4 . For example, the $\mathrm{m} / \mathrm{z}$ of the ion may be too high for the RF amplitude available for the quadrupole to reach $q \geq 0.4$ or, perhaps, the well-depth at high $q$ values is too deep for the amplifiers producing the mixed FM waveform to output sufficient power to eject the ions. Another possibility is that the untargeted ions being removed are labile and undergo fragmentation while being excited by the mixed FM waveform. Fragment ions produced in this manner might be left behind after the isolation period. All of these problems can be mitigated by positioning the ion to be isolated at $q<0.4$. At these $q$-values, the maximum $q$ value accessible by the mixed FM waveform decreases and the $\mathrm{m} / \mathrm{z}$ range that fails to be eliminated expands. For example, if the ion to be isolated is placed at $q=0.3$, the low $\mathrm{m} / \mathrm{z}$ cutoff ( $q=0.908$ ) corresponds to $33 \%$ of the $\mathrm{m} / \mathrm{z}$ of the ion being isolated, and the lowest $\mathrm{m} / \mathrm{z}$ that the mixed FM waveform is able to remove is $39 \%$ of the $\mathrm{m} / \mathrm{z}$ of the ion being isolated. Ions with $\mathrm{m} / \mathrm{z}$ between $33 \%$ and $39 \%$ of the ion being isolated will not be removed from the quadrupole. Figure $4 \mathrm{~b}$ shows a plot depicting the minimum $\mathrm{m} / \mathrm{z}$ that is removed by the mixed FM waveform and the maximum $\mathrm{m} / \mathrm{z}$ that is removed by the low $\mathrm{m} / \mathrm{z}$ cutoff as a function of the $q$-value of the ion being isolated. This plot shows the expansion of the stable region (i.e., the $\mathrm{m} / \mathrm{z}$ range that is not removed either by the mixed FM waveform or the low $\mathrm{m} / \mathrm{z}$ cutoff) as $q$ is decreased. Figure $4 \mathrm{~b}$ also graphically demonstrates that the stable region is quite small when $q \geq 0.4$.

Although Figure $4 \mathrm{~b}$ makes it seem as though it is desirable to use a $q$-value as near to 0.4 as possible, there is one advantage when going to low $q$-values: although the $\mathrm{m} / \mathrm{z}$ range that 
cannot be eliminated expands, the lowest $\mathrm{m} / \mathrm{z}$ value that can be reached with the mixed FM waveform decreases. For example, if the ion being isolated $(\mathrm{m} / \mathrm{z}=1000)$ is positioned at $q=0.3$, the low $\mathrm{m} / \mathrm{z}$ cutoff is 330 and the mixed FM waveform can reach 389 . But if $\mathrm{m} / \mathrm{z}=1000$ is positioned at $q=0.2$, the low $\mathrm{m} / \mathrm{z}$ cutoff is 220 and the mixed FM waveform can reach 356 . Therefore if an untargeted peak is present at $\mathrm{m} / \mathrm{z}=370$, it cannot be removed with $\mathrm{m} / \mathrm{z}=1000$ positioned at $q=0.3$ but it can be if $\mathrm{m} / \mathrm{z}=1000$ is positioned at $q=0.2$.

From the above analysis it is apparent that 0.637 is a good choice for the $q$-value of the ion to be isolated, unless there are mitigating circumstances such as those just described, because the bandwidth of the mixed FM waveform is minimized. Lower $q$-values down to 0.4 are also useful because the well depths are smaller making the untargeted ions easier to remove. As discussed above, $q$-values below 0.4 can be used if required, but there will be an increasingly large window of $\mathrm{m} / \mathrm{z}$ that cannot be eliminated as $q$ is decreased. We have little experience with $q$-values above 0.637 because large well depths make elimination of untargeted ions difficult, especially when isolating ions above $1000 \mathrm{~m} / \mathrm{z}$. There is one potential advantage to using high $q$ values, and that is that higher $q$-values place neighboring $\mathrm{m} / \mathrm{z}$ values farther apart in frequency, which might allow higher resolution isolations to be performed.

As an example of the determination of the various frequencies associated with a given isolation, if $q=0.5$ is chosen as a compromise between bandwidth and well depth, equation 1 gives $f_{n}=153 \mathrm{kHz}$ (for $f_{\text {drive }}=816 \mathrm{kHz}$ ) and the calculation for $f_{\text {max }}$ yields $408 \mathrm{kHz}$. The distance from $f_{n}$ to the upper boundary $\left(f_{\max }\right)$ is greater than that between $f_{n}$ and the lower boundary ( $\mathrm{f}=0)$, so in order to reach $f_{\max }$ we have

$$
f_{c}+f_{d}=f_{\max }-f_{n}=255 \mathrm{kHz}
$$


A choice must be made regarding the width of the notch, $\Delta f_{\text {notch }}$, which determines the resolution of the isolation. A notch-width of $2 \mathrm{kHz}$ has been found empirically to be a good starting point, so that

$$
\Delta f_{\text {notch }}=2\left(f_{c}-f_{d}\right)=2 \mathrm{kHz}
$$

The two equations 7) and 8) can be solved for the two unknowns $f_{c}$ and $f_{d}$, which turn out to be $f_{c}=128 \mathrm{kHz}$ and $f_{d}=127 \mathrm{kHz}$ in this example. It should be noted that the above procedure for calculating $f_{c}$ and $f_{d}$ is only valid for $0.449<q<0.637$. For $q>0.637$ instead of equation 7) we have

$$
f_{c}+f_{d}=f_{n}
$$

so that the lower sideband extends to $f=0$. For $q<0.449$ the folding problem must be recognized to ensure that $f_{c} \leq f_{n}$ rather than using equation 7) or 9). For the remaining parameter $f_{m}, 10 \mathrm{~Hz}$ has been found empirically to provide a signal spacing that enables elimination of untargeted ions throughout the bandwidth of the mixed FM waveform. It is expected that $f_{m}$ may vary in proportion to $f_{\text {drive }}$, since $f_{\text {drive }}$ sets the width of the frequency band occupied by ions within the quadrupole.

Performance of the Mixed FM Waveform. Figure 5 demonstrates application of the mixed FM waveform for isolation of various peaks from Agilent ESI tuning mix for ion traps. The pre-isolation spectra are shown in panels A-C and the corresponding post-isolation spectra 
are shown in panels D-F. For these experiments, the ion to be isolated was positioned at $q=0.5$, with the exception of $\mathrm{m} / \mathrm{z}=323$ which was positioned at $q=0.636$. The width of the frequency notch shrank from 2.6 to $1.14 \mathrm{kHz}$ as $\mathrm{m} / \mathrm{z}$ increased. The mixed FM waveform was applied for $300 \mathrm{~ms}$ for $\mathrm{m} / \mathrm{z}=323,623$, and 923 . The time was increased to $1000 \mathrm{~ms}$ for the higher $\mathrm{m} / \mathrm{z}$ ions.
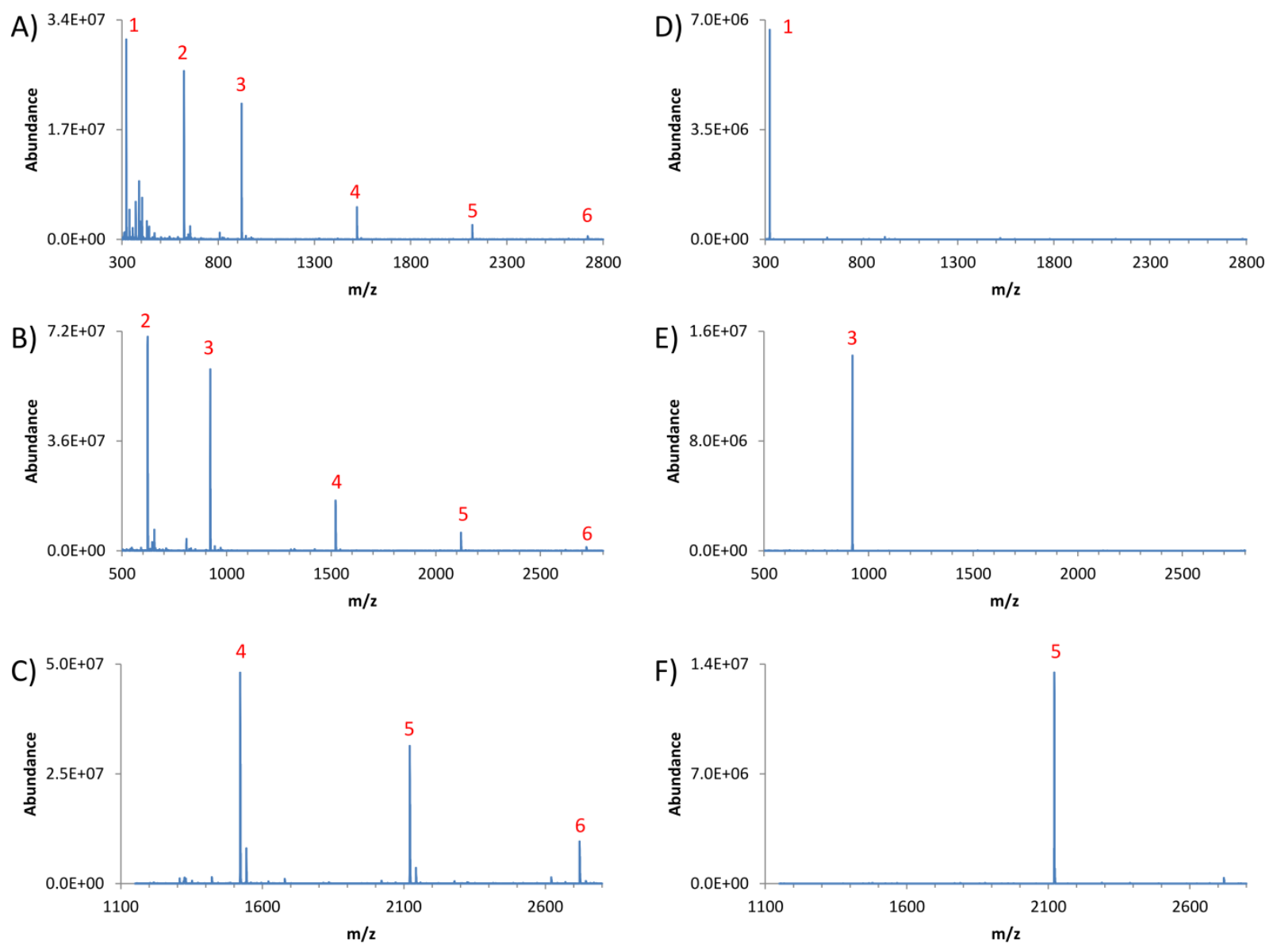

Figure 5. Demonstration of the mixed FM technique for isolating various ions in Agilent ESI tuning mix. This sample includes: (1) hexamethoxyphosphazene; (2) hexakis(2,2difluoroethoxy)phosphazene; (3) hexakis $(1 \mathrm{H}, 1 \mathrm{H}$, 3H-tetrafluoropropoxy)phosphazene; (4) hexakis $(1 \mathrm{H}, \quad 1 \mathrm{H}, \quad 5 \mathrm{H}$-octafluoropentoxy)phosphazene; $\quad(5)$ hexakis $(1 \mathrm{H}, \quad 1 \mathrm{H}, \quad 7 \mathrm{H}-$ dodecafluoroheptoxy)phosphazene; and (6) hexakis $(1 \mathrm{H}, \quad 1 \mathrm{H}, \quad 9 \mathrm{H}-$ perfluorononyloxy)phosphazene. Pre-isolation spectra are shown in A-C while corresponding post-isolation spectra are shown in D-F. 
Figure 6 compares the resolution and efficiency of isolation using the mixed FM waveform to two other isolation methods that are available on our instrument (Q1 mass filter and apex isolation in Q3). The spectra show that the mixed FM waveform exhibits efficiencies similar to apex isolation in Q3 when the two techniques are adjusted for unit mass resolution. Isolation using Q1 as a mass filter significantly underperforms to the point that we were not able to collect data for the higher $\mathrm{m} / \mathrm{z}$ ions because of insufficient signal as resolution approached unit mass. As discussed in the introduction, however, trapping ions prior to isolation requires additional time. While apex isolation and the mixed FM waveform both require prior trapping and cooling of the ions, isolation can be performed faster using the apex. This is evidenced by the fact that the mixed FM waveform was applied for either $300 \mathrm{~ms}(\mathrm{~m} / \mathrm{z}=323$ and 923) or 1000 $\mathrm{ms}(\mathrm{m} / \mathrm{z}=2122,2722)$ while collecting the data in Figure 6, but apex isolation was performed in only $3 \mathrm{~ms}$.
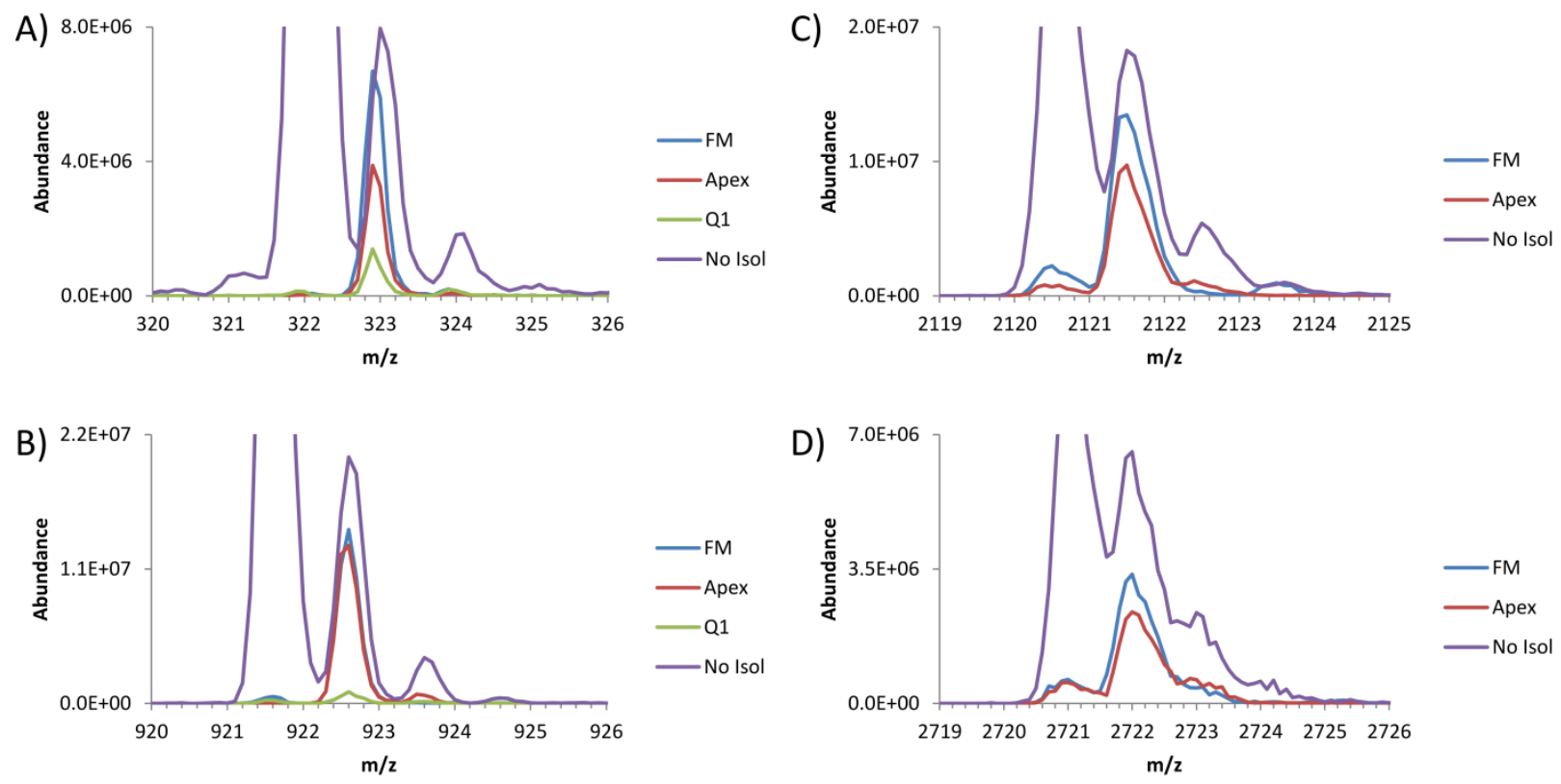

Figure 6. Comparison of isolation using the mixed FM waveform in Q3, apex isolation in Q3, and Q1 as a mass filter. Mixed FM and apex isolation exhibit similar efficiency when operated at 
unit mass resolution. Data was not acquired for the two higher $\mathrm{m} / \mathrm{z}$ ions with the Q1 mass filter because of insufficient signal intensity.

Figure 7 shows spectra produced after attempting to isolate $m / z=923$ by applying the mixed FM waveform for different lengths of time. Insufficient time results in incomplete removal of untargeted ions whereas excessive time results in loss of target ions as demonstrated by comparing abundances in Figure 7c and Figure 7d. The mixed FM waveform must be applied for a length of time sufficient for the untargeted ions to absorb enough energy to be ejected from the trap. This time is dependent upon the amplitude of the mixed FM waveform, the overlap between the ion's absorption band and the signals making up the mixed FM waveform, and the cooling rate due to collisions with background gas molecules. Additionally, sampling theory imposes limits on the exposure time. For example, if one wishes to resolve ions with secular frequencies $81 \mathrm{~Hz}$ apart, as is the case in Figure 6c, the exposure time must be at least $2 \times(1 /$ $81)=25 \mathrm{~ms}$. These limitations are inherent in any isolation method that involves application of multi-frequency waveforms. 

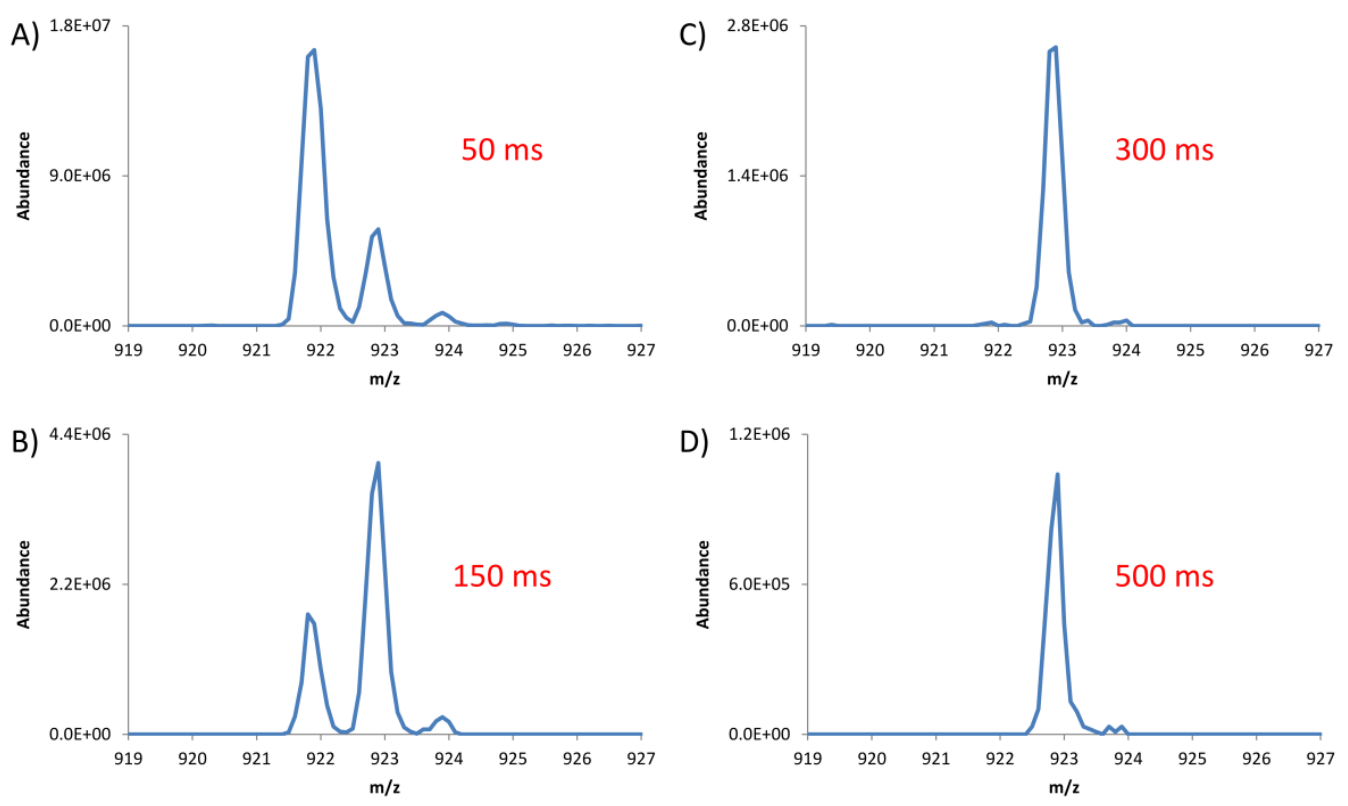

Figure 7. Spectra acquired after attempting to isolate $\mathrm{m} / \mathrm{z}=923$ (hexakis $(1 \mathrm{H}, 1 \mathrm{H}, 3 \mathrm{H}-$ tetrafluoropropoxy)phosphazene) by application of the mixed FM waveform for various lengths of time. The waveform must be applied for a time sufficient for the untargeted ions to be ejected from the trap; however, applying the waveform for too long results in loss of target ions.

One advantage of the mixed FM technique over apex isolation and quadrupole mass filters is that the mixed FM waveform works over a broader $\mathrm{m} / \mathrm{z}$ range. Higher $\mathrm{m} / \mathrm{z}$ values require higher RF and DC potentials to place them at the tip of the stability diagram. Therefore, the $\mathrm{m} / \mathrm{z}$ range is limited by the RF and DC power supplies. With the mixed FM waveform, the power supplies limit the $q$-value at which the ion to be isolated may be placed. However, low $q$ values are often sufficient for isolation of high $\mathrm{m} / \mathrm{z}$ ions. Low $q$-value is even a requirement when isolating certain high $\mathrm{m} / \mathrm{z}$ ions because the lower pseudopotential well depth means less heating occurs during isolation. Reduction of heating is often necessary to minimize fragmentation of the target ions. Figure 8 demonstrates isolation of a single species formed from the ion/ion reaction between sodiated polyethylene glycol ions $\left[\mathrm{PEG}(\mathrm{n}=72-80)+3 \mathrm{Na}^{+}\right]^{3+}$ and carborane anion $\left(\mathrm{CHB}_{11} \mathrm{Cl}_{11}\right)^{-}$. The isolated peak corresponds to $\left[\mathrm{PEG}(\mathrm{n}=76)+3 \mathrm{Na}^{+}+\right.$ 
$\left.2\left(\mathrm{CHB}_{11} \mathrm{Cl}_{11}\right)^{-}\right]^{+}$, with $\mathrm{m} / \mathrm{z}=4479$. This $\mathrm{m} / \mathrm{z}$ is well beyond the range accessible via apex isolation on the QTRAP 4000, which is limited to $\mathrm{m} / \mathrm{z}=3000$.
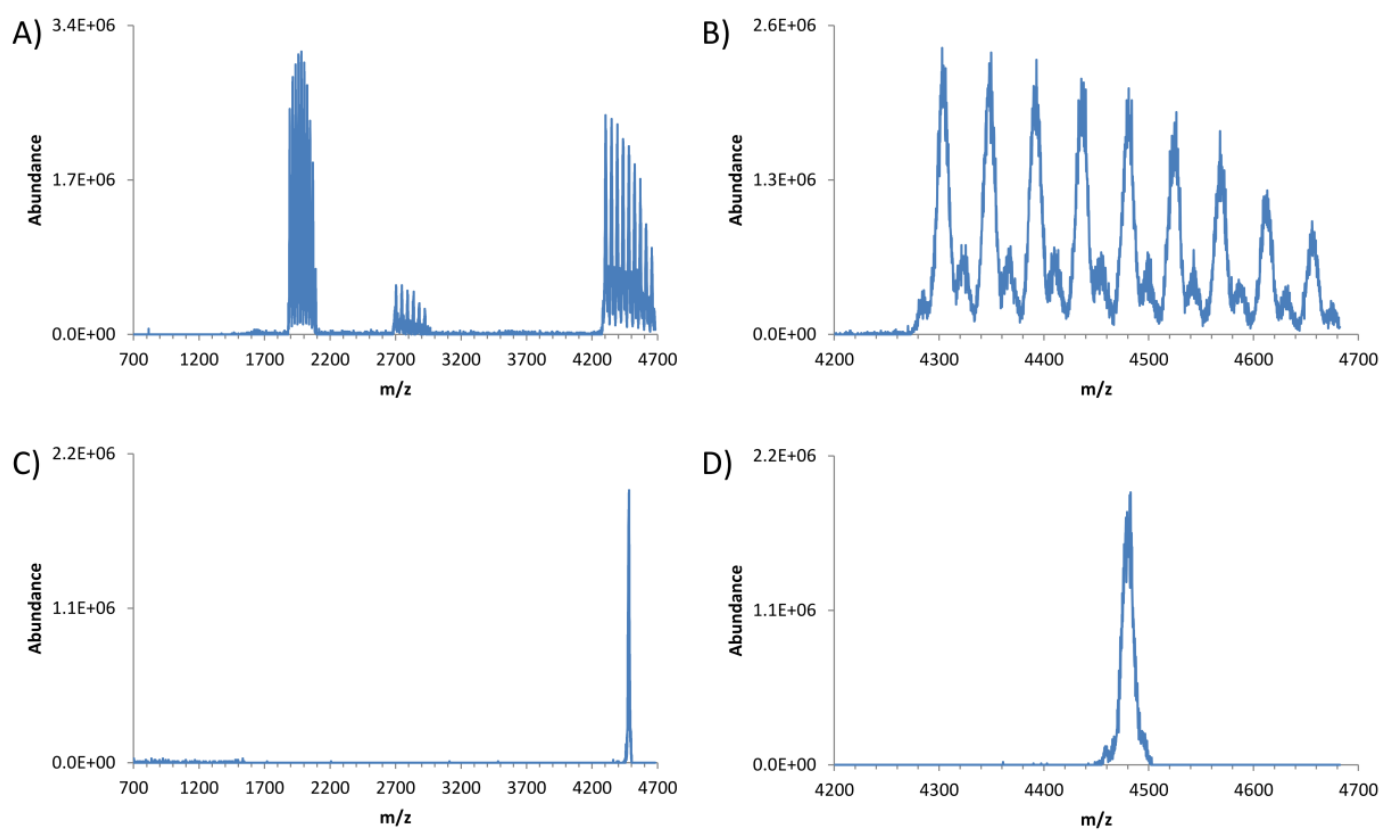

Figure 8. Application of the mixed FM waveform for isolation of a high $\mathrm{m} / \mathrm{z}$ ion $[\mathrm{PEG}(\mathrm{n}=76)+$ $\left.3 \mathrm{Na}^{+}+2\left(\mathrm{CHB}_{11} \mathrm{Cl}_{11}\right)\right]^{+}$that was produced by the ion/ion reaction between sodiated PEG ion and the carborane anion (see text). Pre-isolation spectra are shown in A) and B); post-isolation spectra are shown in $\mathrm{C}$ ) and $\mathrm{D}$ ). The pre-isolation spectrum of $\mathrm{A}$ ) shows a series of doubly charged PEG ions of the form $\left[\mathrm{PEG}(\mathrm{n}=72-80)+3 \mathrm{Na}^{+}+\left(\mathrm{CHB}_{11} \mathrm{Cl}_{11}\right)^{-2+}\right.$, a series of shorter chain PEG ions of the form $\left[\mathrm{PEG}(\mathrm{n}=48-53)+2 \mathrm{Na}^{+}+\left(\mathrm{CHB}_{11} \mathrm{Cl}_{11}\right)^{-}\right]^{+}$that were present in the cationic reactant population (see ions at roughly $\mathrm{m} / \mathrm{z} 2700-3000$ ), and a series of singly charged ions of the form $\left[\mathrm{PEG}(\mathrm{n}=72-80)+3 \mathrm{Na}^{+}+2\left(\mathrm{CHB}_{11} \mathrm{Cl}_{11}\right)\right]^{+}$. The pre-isolation spectrum of $\left.\mathrm{B}\right)$ is a blow-up of the latter ion population. The ion to be isolated was positioned at $q=0.1$ and the mixed FM waveform was applied for $300 \mathrm{~ms}$.

\section{CONCLUSIONS}

We have demonstrated ion isolation in a quadrupole ion trap using a new method for generating a tailored waveform called the mixed FM technique. The technique involves generation of a broadband waveform with a frequency notch within which the ion to be isolated is positioned. The choice of $q$-value at which to position the ion to be isolated is important as it 
determines the secular frequencies of all ions. At $q$-values above about 0.449 , all contaminating ions may be removed using the mixed FM technique. The lower boundary extends to $q=0.4$ if one allows for a very small window of inaccessible $\mathrm{m} / \mathrm{z}$ space. Although this space expands as $q$ is decreased below 0.4 , this may be necessary in order to remove untargeted ions at low $\mathrm{m} / \mathrm{z}$ values or prevent fragmentation. At a $q$-value of about 0.637 , the smallest bandwidth is required for the mixed FM waveform. Relative to other tailored waveform methods for ion isolation in ion traps, the present approach is less flexible than those in which waveforms are calculated and downloaded to a frequency synthesizer. However, the present approach is relatively simple to implement and can be readily tuned by simply turning a knob on a function generator without recourse to a new calculation and download.

The mixed FM technique demonstrated performance similar to that of apex isolation with respect to resolution and efficiency. A disadvantage of the mixed FM technique (and similar techniques involving broadband waveforms) is that it requires more time than apex-based methods. However, the mixed FM technique can be applied to a much larger range of $\mathrm{m} / \mathrm{z}$ because it is not as limited by the RF and DC power supplies driving the quadrupole.

\section{ACKNOWLEDGEMENTS}

We would like to thank Dr. Steven Cummings and Prof. Christopher A. Reed of the University of California - Riverside for synthesizing the carborane salt. This work was supported by AB

Sciex (RLH) and Office of Basic Energy Sciences, Office of Science, U.S. Department of Energy, under Award DE-FG02-00ER15105 (CAL and BMP). 


\section{REFERENCES}

1 Gross, J. H. Mass Spectrometry: A Textbook, 2nd ed.; Springer: New York, 2011; pp 415478.

2 Gross, J. H. Mass Spectrometry: A Textbook, 2nd ed.; Springer: New York, 2011; pp 146152.

3 March, R. E.; Todd, J. F. J. Practical Aspects of Ion Trap Mass Spectrometry, vol. III; CRC Press: Boca Raton, 1995; pp 39-42.

4 Kenny, D. V.; Callahan, P. J.; Gordon, S. M.; Stiller, S. W. Rapid Commun. Mass Spectrom. 1993, 7, 1086-1089.

5 Goeringer, D. E.; Asano, K. G.; McLuckey, S. A.; Hoekman, D.; Stiller, S. W. Anal. Chem. 1994, 66, 313-318.

6 Marshall, A. G.; Wang, T.-C. L.; Ricca, T. L. J. Am. Chem. Soc. 1985, 107, 7893.

7 Cody, R. B.; Hein, R. E.; Goodman, S. D. Rapid Commun. Mass Spectrom. 1987, 1, 99102.

8 Juhasz, M.; Hoffmann, S.; Stoyanov, E.; Kim, K. C.; Reed, C. A. Angew. Chem., Int. Ed. 2004, 43, 5352-5355.

9 Xia, Y.; Wu, J.; Londry, F. A.; Hager, J. W.; McLuckey, S. A. J. Am. Soc. Mass Spectrom. 2005, 16, 71-81.

10 Van Berkel, G. J.; Asano, K. G.; Schnier, P. D. J. Am. Soc. Mass Spectrom. 2001, 12, 853-862.

11 Londry, F. A.; Hager, J. W. J. Am. Soc. Mass Spectrom. 2003, 14, 1130-1147.

12 Liang, X.; Xia, Y.; McLuckey, S. A. Anal. Chem. 2006, 78, 3208-3212.

13 Koizumi, H.; Whitten, W. B.; Reilly, P. T. A.; Koizumi, E. Int. J. Mass Spectrom. 2009, 286, 64-69. 
$f_{c}=50 \mathrm{kHz}$ modulated from $1 \mathrm{kHz}$ to $99 \mathrm{kHz}\left(f_{d}=49 \mathrm{kHz}\right)$

with a frequency of $f_{m}=10 \mathrm{~Hz}$

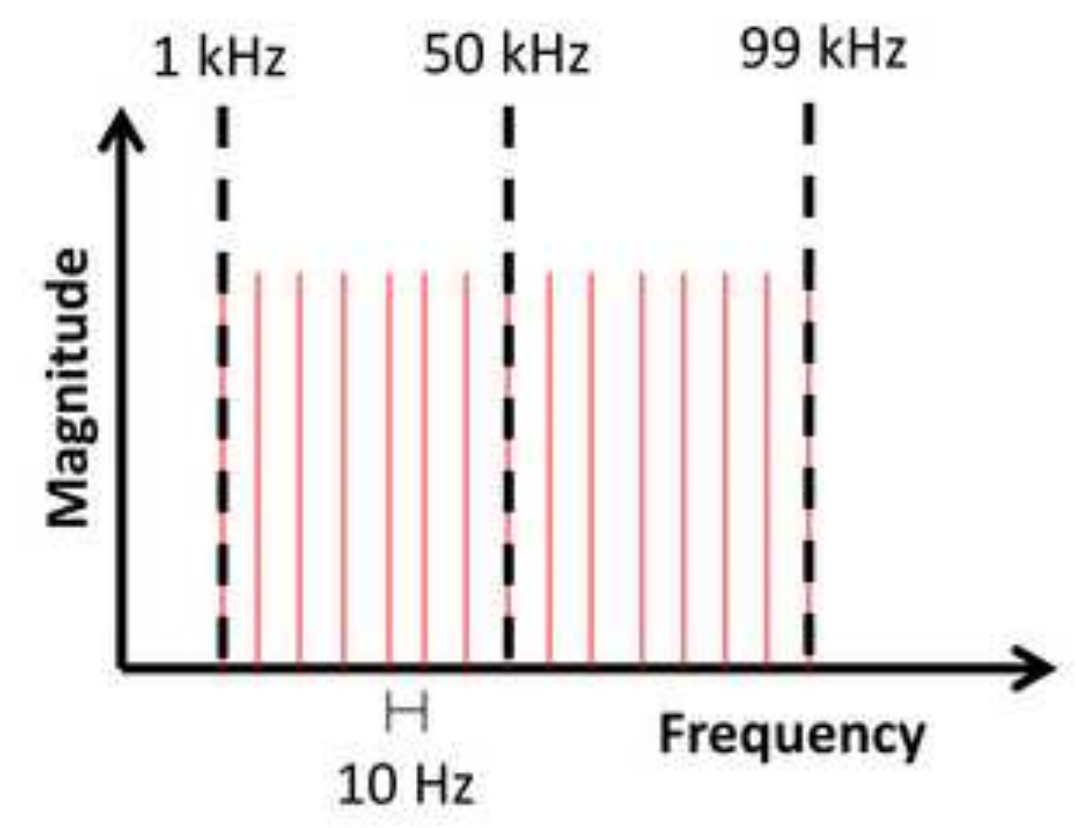

Untargeted ions

go here
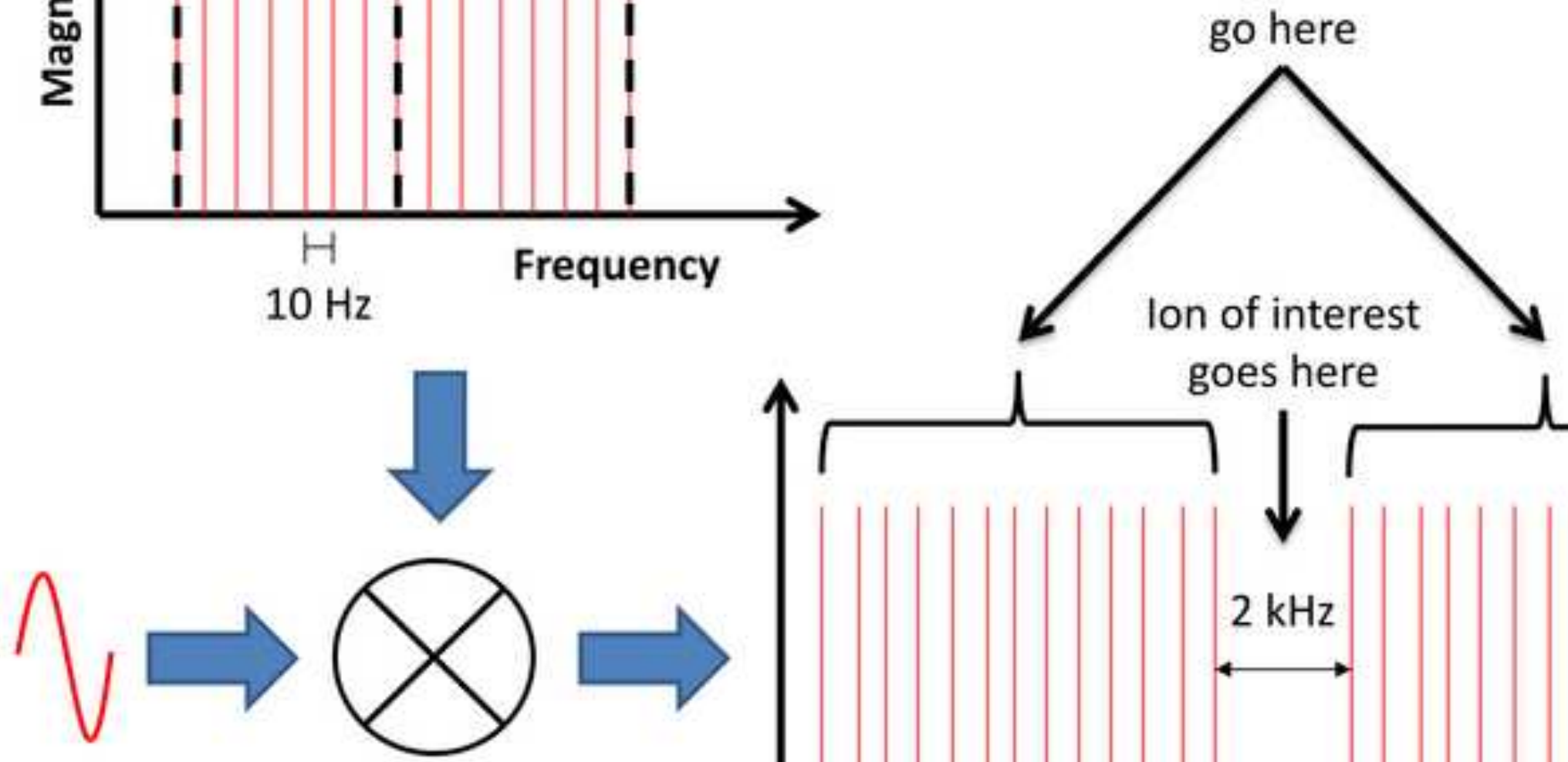

Sine wave at target's resonant frequency $f_{n}$

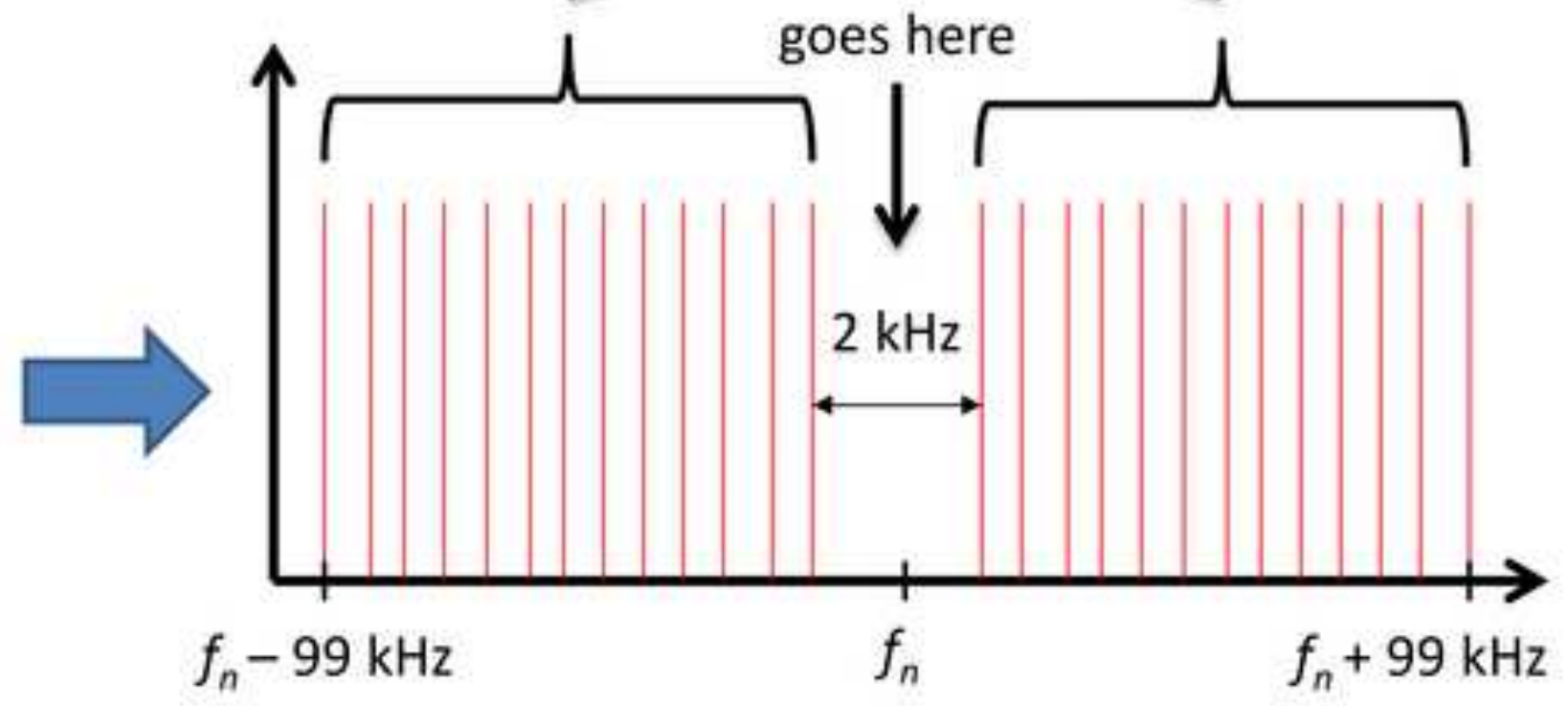

